\title{
Tuberculosis as a Cause of Broncho-esophageal Fistula
}

\author{
Thara Pratap, Muhammed Jasim Abdul Jalal' ${ }^{1}$, Hari Lakshmanan P², A. K. Vishnu, Antony Paul Chettupuzha ${ }^{3}$ \\ Departments of Radiology, 'Internal Medicine and Rheumatology, ${ }^{2}$ Pulmonology and ${ }^{3}$ Medical Gastroenterology, VPS Lakeshore Hospital, Kochi, Kerala, India
}

\section{Abstract}

We report a case of broncho-esophageal fistula associated with tuberculosis. A 29-year-old female diagnosed with smear-positive pulmonary tuberculosis on anti-tuberculosis treatment for 2 months presented with complaints of cough on food intake with food particles present in the expectorant. Radiological examination revealed mediastinal lymphadenopathy and upper gastrointestinal endoscopy revealed multiple fistulous tracts in the esophagus. Small fistulae were treated by clipping and large fistulae were treated with self-expandable metallic stents which resulted in the alleviation of symptoms.

Keywords: Broncho-esophageal fistula, mediastinal lymphadenopathy, tuberculosis

\section{INTRODUCTION}

The most common cause of broncho-esophageal fistula (BOF) is malignancy. ${ }^{[1]}$ Among the nonmalignant infective cause, tuberculosis is common. ${ }^{[1]}$ In 2002, Lado et al. reported 36 cases of BOF secondary to tuberculosis. The combination of mediastinal adenopathy and cough following food intake hints to the possibility of BOF. Here, we describe a case of tuberculosis causing BOF.

\section{Case Report}

A 29-year-old female was diagnosed with smear-positive pulmonary tuberculosis and was started on anti-tuberculosis treatment. She had clinical and radiological resolution initially. Two months later, she presented with complaints of cough on food intake with food particles present in the expectorant. There was no history of shortness of breath, chest pain, vomiting, or choking. There was also no obvious history of foreign body aspiration, ingestion of toxic or corrosive substances. She also had a past history of infiltrating ductal carcinoma left breast (pT1c[m] N0[0/5]) (estrogen receptor $3+3=6 / 8$; progesterone receptor negative; Her 2 neu positive [score $3+$ ]) for which she had undergone left modified radical mastectomy.

Clinical examination revealed pallor and diffuse crepitations in the right lung fields. There was no icterus, cyanosis, clubbing, or cervical lymph node enlargement. Pulse rate was regular and 96/min; blood pressure was $110 / 70 \mathrm{mmHg}$; the

\begin{tabular}{|l|l|}
\hline \multicolumn{3}{|c|}{ Access this article online } \\
\hline Quick Response Code: & Website: \\
\hline & www.ijrc.in \\
\hline
\end{tabular}

respiratory rate was $20 / \mathrm{min}$, and she was afebrile. Oxygen saturation was $94 \%$ in room air. Other systems were within normal limits.

Investigations revealed normal hemogram with elevated erythrocyte sedimentation rate of $52 \mathrm{~mm} / \mathrm{h}$. The frontal radiograph of the chest [Figure 1] revealed consolidation in the right mid-zone. Sputum for cartridge-based nucleic acid amplification test was done. Rifampicin resistance was not detected. Bronchial secretions tested positive for tuberculosis by polymerase chain reaction.

Contrast-enhanced computed tomography of the thorax [Figure 2] revealed consolidation in the right middle and lower lobes with air bronchogram and evidence of bronchiectasis. Few areas of subpleural consolidation with adjacent fibrosis were also seen in the left lung. Multiple enlarged necrotic lymph nodes with peripheral enhancement were noted in the mediastinum [Figures 3 and 4]. A necrotic lymph node mass in the subcarinal region and right hilar region was seen to be contiguous with the esophagus and bronchus

Address for correspondence: Dr. Muhammed Jasim Abdul Jalal, Department of Internal Medicine and Rheumatology. VPS Lakeshore Hospital, Nettoor (P0) Maradu, NH 47-Byepass, Kochi - 682 040, Kerala, India. E-mail: jasimabduljalal@yahoo.com

This is an open access journal, and articles are distributed under the terms of the Creative Commons Attribution-NonCommercial-ShareAlike 4.0 License, which allows others to remix, tweak, and build upon the work non-commercially, as long as appropriate credit is given and the new creations are licensed under the identical terms.

For reprints contact: WKHLRPMedknow_reprints@wolterskluwer.com

How to cite this article: Pratap T, Jalal MJ, Lakshmanan HP, Vishnu AK, Chettupuzha AP. Tuberculosis as a cause of broncho-esophageal fistula. Indian J Respir Care 2021;10:257-9.

Received: 07-03-2021 Revised: 18-04-2021

Accepted: 16-05-2021 Published: 14-06-2021 
Pratap, et al.: Broncho-esophageal fistula

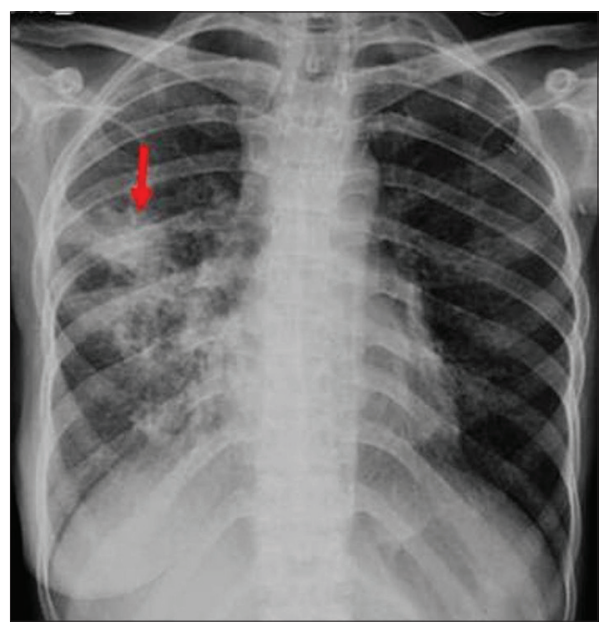

Figure 1: Frontal radiograph of the chest show patchy opacities with air bronchogram in the right lung mid and lower zones when the patient presented with cough on food intake. The left lung appears more translucent (post mastectomy status)

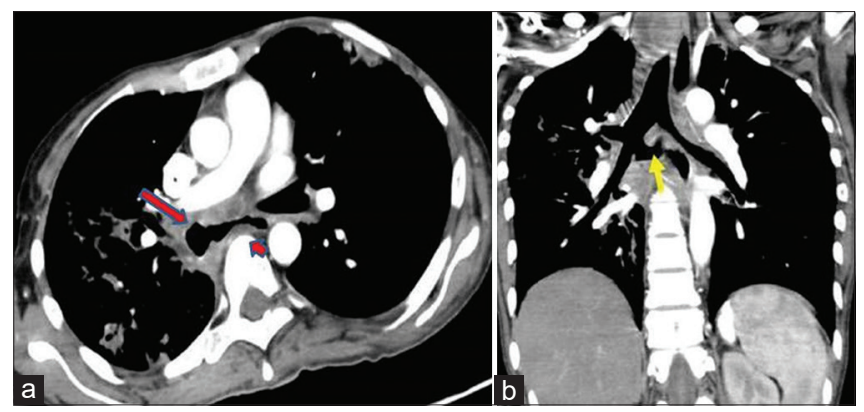

Figure 3: (a) Contrast-enhanced computed tomography thorax in mediastinal window reveal multiple enlarged necrotic mediastinal lymph nodes in the paratracheal region (arrow) with peripheral enhancement. (b) Necrotic lymph node mass in the sub-carinal region was seen to be contiguous with the esophagus and bronchus intermedius with a fistulous communication between the esophagus and bronchus intermedius (arrow)

intermedius with a fistulous communication between the esophagus and bronchus intermedius [Figure 3 and 4].

Upper gastrointestinal endoscopy was done which revealed multiple fistulous tracts in the esophagus [Figure 5]. Virtual bronchoscopy at the level of distal right bronchus showed the fistulous tract [Figure 6]. Small fistulae were treated by clipping and large fistulae were treated with self-expandable metallic stents. The patient was kept on Ryle's tube feed which resulted in the alleviation of symptoms.

Considering the stage of malignancy and aggressive treatment given, the chances of metastasis were negligible. Keeping in mind, the necrotic mediastinal lymphadenopathy in a microbiologically proven case of tuberculosis, the clinical diagnosis of fistula and lymphadenopathy was considered as tuberculosis. Hence, the efforts to take the biopsy of fistulous tract and fine-needle aspiration cytology/biopsy of mediastinal lymphadenopathy were deferred.

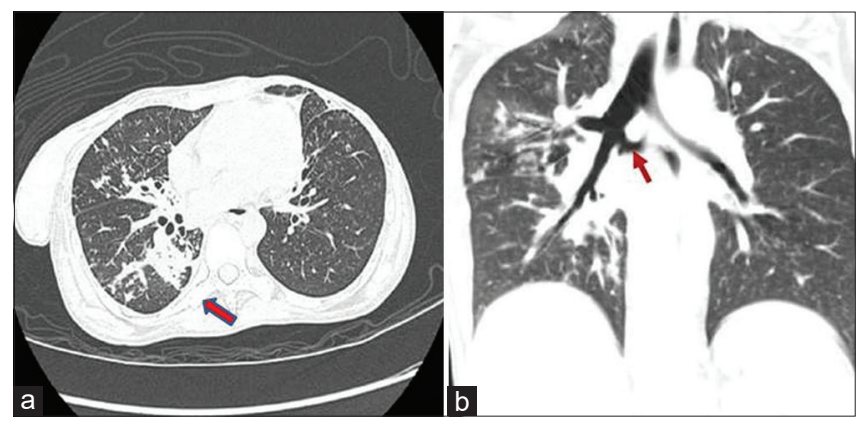

Figure 2: (a) Contrast-enhanced computed tomography thorax axial sections in lung window reveal wedge shaped and patchy areas of consolidation with air bronchogram in the right lung mainly in the anterior segment of upper lobe, middle and predominantly in the apical segment of lower lobe (arrows). (b) Coronal sections showing broncho-oesophageal fistulae (arrows)
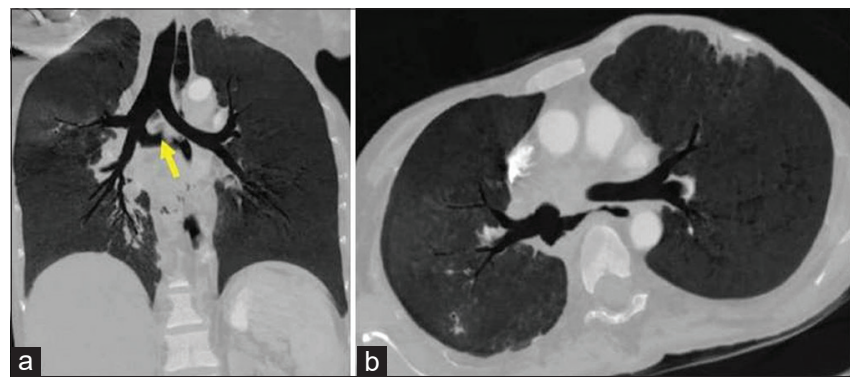

Figure 4 (a and $\mathbf{b}$ ): Contrast-enhanced computed tomography thorax in minimum intensity projection re-demonstrating broncho-esophageal fistula

\section{Discussion}

Noncancerous broncho-esophageal fistula is rare but causes significant morbidity due to recurrent pulmonary infection. A high index of suspicion is the key for early detection. Usual clinical presentation is as paroxysmal cough particularly following food intake. ${ }^{[1-3]}$ The commonest cause of infective nonmalignant $\mathrm{BOF}$ is reported to be mediastinal tuberculosis.

Tuberculous broncho-esophageal fistula is related to mediastinal lymph node involvement which leads to surrounding peri-esophagitis and peri-tracheitis. ${ }^{[1]}$ Subsequent healing with scar formation results in the traction diverticulum of the esophagus.

Mechanisms of tuberculous BOF include: ${ }^{[2]}$

1. Enlargement of mediastinal nodes with inflammatory changes involving the adjacent structures

2. Caseous necrosis of lymph nodes with secondary rupture into the esophagus and bronchus

3. Calcification of the mediastinal nodes with secondary erosion into the esophagus and the tracheobronchial tree results in broncholithiasis

4. Fibrosis around the esophagus secondary to resolving adenitis can produce a traction esophageal diverticulum, which can rupture into the tracheobronchial tree

5. Erosion of the diverticulum and a bronchus by a calcified lymph node. 


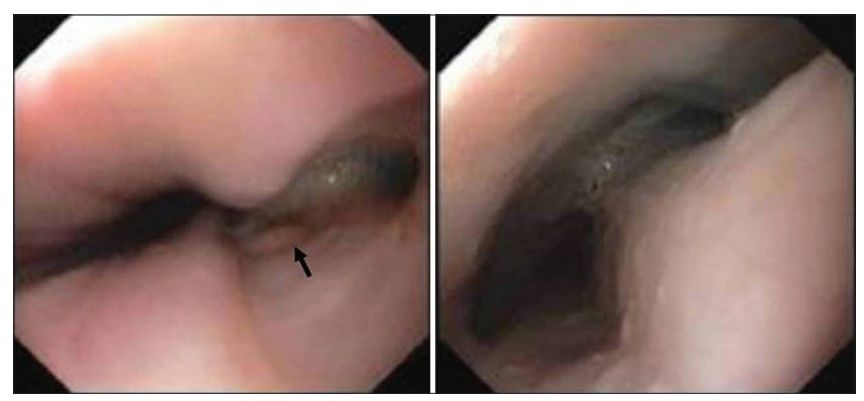

Figure 5: Upper gastrointestinal endoscopy shows fistulous tract between the esophagus and bronchus intermedius

Other benign causes of BOF include trauma, ingestion of foreign body or toxic chemicals, iatrogenic, pressure necrosis by a cuffed tracheostomy or endo-tracheal tube, etc.

Conventional fluoroscopy with nonionic iodinated oral contrast medium is the initial investigation of choice for evaluation of suspected fistulae; however, endoscopy may be needed for definitive diagnosis. ${ }^{[3-5]}$ Contrast-enhanced computed tomography of the thorax can give a clue to the etiology of the fistula while a computed tomography oral contrast swallow study can give a three-dimensional view of the fistula. ${ }^{[3-5]}$

Bronchoesophageal fistula is usually managed surgically. Simple ligation and resection of the fistula can be performed if the fistulous tract originates from lymph nodes with no parenchymal complication. ${ }^{[6]}$ In our case, clipping was done for the fistula and the patient was kept on Ryle's tube feed which resulted in the subsidence of symptoms.

In young healthy patients who can tolerate thoracoscopic maneuvers video-assisted thoracoscopic surgery (VATS) may be an effective and minimally invasive approach.$^{[7]}$ However, VATS is questionable in the case of a large fistula or where isolation of fistula is difficult due to adhesions.

Stenting has become the management of choice in broncho-esophageal fistula since 1990 when Song et al. reported their success with silicone-covered expandable esophageal stents. ${ }^{[8]}$ This lowered the rate of complications and mortality compared to conventional treatment. In most cases, insertion of a single stent, either a tracheobronchial or an esophageal stent, is sufficient to seal off the fistula. If dyspnea or dysphagia is not relieved, a second stent is indicated. When there is airway compression or when the fistula is insufficiently sealed by an esophageal stent and when both the esophagus and airways are severely invaded by malignancy, stenting in both the esophagus and airways should be considered.

\section{Conclusion}

This case highlights the noncancerous causes of BOF, especially tuberculosis which has a high prevalence in India.

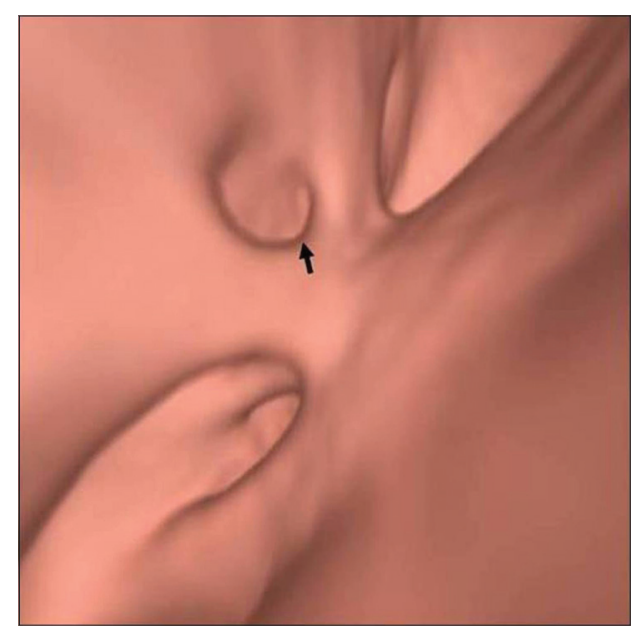

Figure 6: Virtual bronchoscopy at the level of distal right bronchus showing the fistulous track (arrow) in addition to origin of right upper lobe bronchus and bronchus intermedius

It also highlights endoscopic procedure an alternate treatment modality for BOF, especially in a poor surgical candidate.

\section{Declaration of patient consent}

The authors certify that they have obtained all appropriate patient consent forms. In the form, the patient has given her consent for her images and other clinical information to be reported in the journal. The patient understands that name and initials will not be published and due efforts will be made to conceal identity, but anonymity cannot be guaranteed.

\section{Financial support and sponsorship}

Nil.

\section{Conflicts of interest}

There are no conflicts of interest.

\section{ReFERENCES}

1. Narayanan S, PV Shiji, Majeed KA, V Udayabhaskaran. Tuberculosis presenting as bronchoesophageal fistula. ID Cases 2017;8:19-21.

2. Martins A, Gonçalves Á, Almeida T, Fazeres F, Midões A. Bronchoesophageal fistula in the setting of tuberculosis infection. J Gastrointest Surg 2017;21:2119-21.

3. Fernández Gajardo J, Gómez Herrero H, Viteri Ramírez G. Long-term acquired bronchoesophageal fistula. Arch Bronconeumol 2018;54:283.

4. Barbara DW, Broski SM, Blackmon S. Bronchoesophageal fistula. Can J Anaesth 2017;64:1267-8.

5. Argüder E, Aykun G, Karalezli A, Hasanoğlu HC. Bronchoesophageal fistula. J Bronchology Interv Pulmonol 2012;19:47-9.

6. Indiran V. Tuberculous bronchoesophageal fistula presenting as intractable cough. Tuberk Toraks 2017;65:60-2.

7. Ginesu GC, Feo CF, Cossu ML, Ruiu F, Addis F, Fancellu A, et al. Thoracoscopic treatment of a broncho-esophageal fistula: A case report. Int J Surg Case Rep 2016;28:74-7.

8. Wang CY, Chou CH, Wang HP, Chen JS, Lee P. Successful treatment of bronchoesophageal fistula with esophageal and bronchial stenting. J Formos Med Assoc 2011;110:270-2. 